actual for what is apparent; and in correcting the data of natural by those of artificial pathology. The study of the effects of compound fracture, viewed as a cause of local inflammation and febrile movement in the system, may be considered as the investigation of the effects of a great experiment in man; of pure inflammation from known causes. But in this instance we do not learn more than a series of symptoms and an order of their succession. To discover the intimate nature of the alliance of this known cause to its effects; of the known cause of local inflammation to local and general phenomena, calls for other investigations which can only be followed out by a repetition of the accident, by making the known caúse, the compound fracture, operate in animals through wose transparent tissues the effects on the circulation can be traced, and the influence of the nerves upon it studied and reduced to laws, as done in the foregoing pages. (Prop : IX.)

The contributions made by this method to the science of inflammation stand alone, on account of the certainty of the facts, and form, as it were, a pure pathology.

That a shock acts upon the ganglionic system of nerres, and thus empties the capillary vessels, has been already proved; but what relation the nerrous shock and the exsanguine condition of parts bears to the above group of symptoms, observed as constituting the state of collapse in man, has yet to be enquired into.

One great test of this question is afforded by the effects of loss of blood. For, in whatever way the capillary tubes are rendered bloorless in a healthy sabject, the general results offer features of resemblance, especially in those effects which are referrible to suspension of the capillary functions. For, in regard to this point, it differs little for the time being whether the capillaries are made void by blood flowing ont of the system, or by its retention in the larger veasels of the body. Whatever symptoms therefore are of doubtful origin in collapse may be referred to their true cause by comparison with the symptoms which are attendant on loss of blood. If they are found to exist in both examples, the cause being recognisable in the one, it may justly be inferred in the other.

\section{CASE OF TRAUMATIC TETANUS.} By Jayes Ingris, M.D., Halifax.

(Continued from page. 114.)

POST-MORTEN BXAMINATION FORTY-RIGHT HOURS AFTER DEATH.

Bxtermal aspect.-Body muscular and well formed; pupils; dilated the muscles throughout, which at death were in a state of complete relaxation, are now rigid; there is more lividity of the pending part of the body than is usually seen after the less violent forms of death ; the ammoniacal application to the cervical region had vesicated, and the gunjah plaster adheres firmly to the blistered surface; there is no trace of the abeorbents along the arm.

Head.-On removing the scalp, the temporal muscles are found unusually soft und congested ; the ressels of the dura mater, necessarily torn on opening the craninm, pour forth a quantity of dark fluid blood, with which the larger vessels are also distended ; there is considerable serous effusion in the cavity of the arachnoid, and at the base of the brain, which is continuous along the spine; at the upper and back part of the anterior lobe of the left hemisphere, the serous surfaces of the arachnoid adhere closely together, there is a recent soft granular deposit, with lymph effused; the arachnoid membrane, and the pia-mater, throughout their whole extent, are highly vascular; the structure of the brain itself appears normal, if there be any change the gray matter is perhaps a little darker than usual.; there is no serous effusion in the ventricles, but in that portion of the pia-inater, extending into the inferior cornua ; in the choroid membrane there is a pulpy mass of vesicles, or hydatids, together with an osseo-calcareous deposit, of about the size of a small pea; a similar deposition is found in each choroid membrane; the structure of the cerebellum, like that of the brain, is natural; its pia-matral covering is congested throughout.

Cervical spine.-On removing the spinous processes of the cervical vertebræ, the dura-matral covering of the cord is found in a high state of vascularity; there is considerable effusion in the serous membrane, and the pia-mater is equally congested with the other two membranes; the nervous sheaths passing off from the spine are in a similar state; there are no ossific deposits discoverable; the cord, in substance and appearance, is normal.

We were not permitted to proceed further with the examination of the spine, and with difficulty got consent to make a very partial and hasty examination of the

Abdomen.-Lirer much congested with dark fluid blood, its under surface, as is usually the case, of a yellowish-green colour; size natural; kidneys very large, dark, and congested like the liver; they are softened and indicate structural disease ; urinary bladder contains some fluid; the mucous membrane of the stomach perfectly healthy, and not in the slightest degree vascular; mucous membrane of the colon coated with a healthy secretion of mucus, having the decided green tinge of the extract of gunjah. The examination was here ordered to be stopped.

Chest.-Not examined.

Remarks.-An injury of a similar nature to that which caused tetanus, in the case just given, has lately come under my notice : the finger was saved, and no untoward symptoms followed. It is evident, then, that a predisposing canse must exist; an idiosyncrasy we may call it, by the which one man is subject, after a slight injury, to tetanic irritation; whilst a second, after a similar injury, escapes such a consequence; and a third may receive, with immunity, an injury of a far more serious local character. Post-mortem examinations alone can display to us the cause or causes of such peculiarities; and as there is scarcely a medical man in practice who has not seen, or bad under his own care, a fatal case of tetanus, the cause of this disease might not long remain in obscurity, if, on every future occasion, medical men would urge the necessity of examinations, and then take the trouble to make known to their brethren the appearances discorered.

In almost all the cases of which I have read, and in the few which I have seen, there have been found secreted, upon some portion or other of the investing membranes of the nervous centres, small patches of 
bone, or, a gritty granular deposition, of greater or less solidity, according to the period of its secretion. In the case referred to from the Lancet, there were found " several plates of bone upon the free arachnoid, chiefly opposite the seventh dorsal vertebra;" the arachnoid also, above that rertebra, presented "a minutely granular appearance ;" and, in the case which $I$ have just reported, there were found in the choroid membranes, concrete substances, to a considerable extent. Such abnormal appearances indicate previous irritation in those portions of the membrar.es where such depositions are found, and doubtless a susceptibility to be acted upon again by a slight exciting cause. Now, in an injury such as that which in this instance brought on the tetanic irritation, the extremities of the nerves of sensation and motion, with their accompanying filaments from the ganglionic system, invested by their common membranes, are crushed and mutilated; the result is inflammatory action; and, upon examination, they are found " expanded in bulk, with increased vascularity," as Mr. loodsir discovered in the case related by $\mathrm{Mr}$. Miller, of Edinburgh. In tetanus, the absorbents are never, or only accidentally, found affected; it is not necessary, therefore, as in hydrophobia, that an irritating poison should be absorbed, or that any morbid secretion thrown out at the seat of injury, should be reabsorbed; on the contrary, in tetanus, the irritation commences in the cerebral or spinal membranes, almost as soon as the inflammatory action has begun at the injured extremities of the nerves; and this we can readily understand, from the intimate union of the sympathetic nerve with the regular spinal nerves throughout every portion of the animal machine. This transposition of irritation, we know, takes place to a great extent in many other instances, and we call it "sympathy." But this sympathy seems to exist chiefly between organs of a similar structure, or between portions of the same, or similar, tissues; and thus, in the case before us, the membranous sheaths of the injured extremities of the nerves are the parts which suffer most from the injury, they being most highly vascular; and the membranous coverings of the nervous centres, from previous susceptibility, immediately assume a similar condition, and thence arise the tetanic phenomena.

I think there can be but little doubt with respect to the inflamed membranes of the brain and spinal cord being the seat of this disease; for, although these membranes, intimately connected as they are with the brain or cord, are in a state of the greatest vascularity, and although inflammatory action, has, in many instances, even gone on to granular deposition and effusion of lymph, as in the present case, still the mind is unaffected-the brain continues to perform its office healthily, until checked by pressure from serous effusion; and after death, its structure is found unchanged and normal.

At the invasion of the disease, the muscles of the neck and back are thrown into spasmodic action, probably by the excitation to the cord itself, produced by the inflamed and consequently thickened theca. If inflammatory action be not here arrested, it extends along the sheaths of the regular nerves, passing off from the spine; and the symputhetic nerve becomes of necusity involved; then supervenes that general increased irritability-that peculiar sensibility to external impressions, which is always sooner or later observed in tetanic cases. This increased irritability is concomitant with increased capillary action, and hence we always have warmth of the skin, and often profuse perspiration at this, which may be called the middle, stage of the disease. Should the patient survive this stage, symptoms of serous effusion into the arachnoid gradually appear; respiration and circulation become impeded, or altered; the cold damps of death collect upon the face and bead; and at length the patient passes from off the stage of his human sufferings, perhaps in a convulsive paroxysm, which to the byestanders may appear a struggle of agony, but of which the dying man himself is happily unconscious.:

\section{CASE OF EMPHYSEMA OCCURRING IN}

\section{CHILD-BIRTH.}

By Francis Davies, Surgeon, Pershore.

On Dec. 10th, 1829, at six p.m, I was requested to.see Mrs. C., a delicate lady, with her first child, seven miles in the country. She was complaining of slight pains in the back; discharge trifling; the os uteri but very little dilated; had no rest all night; the pains were not at all improving, nor did the labour appear to be at all advanced; I left her, and returned home.

11th, two p.m. I was again sent for, and found her nearly in the same state; at eight p.n. I gave her forty minims of tiacture of opium; she slept soundly all night, anc was quite well next morning.

12th, eight p.m. I was sent for in a great hurry ; she was represented as being very ill indeed; I found the pains coming on regularly, but still they were slight; the os uteri dilated to the size of half-a-crown, and the edges quite thin, with a good deal of thick glairy mucous discharge, and the patient straining violently, with the idea of soon finishing the labour, as the nurse had told her to be sure to " help her pains." I explained to her the inutility of so doing, and, indeed, the mischief that was likely to ensue, as she was wasting her strength in unnecessary exertion. The labour went on favourably till five a.m., when she was put to bed of a fine boy; the hæmorrhage was very considerable, and $I$ had some difficulty in restraining it. In about a quarter of an hour after the placenta had been discharged, she complained of great difficulty of breathing, and I was astonished at seeing her face, throat, and breast, swollen to three times their natural size, and of a bright scarlet colour. She also complained of great pain in the throat, about two inches above the sternum, and the difficulty of breathing seemed rapidly to increase; indeed, so much so, that she said her breath did not descend lower than the top of her chest. On putting $m y$ hand on the throat, I rery distinctly felt a crackling, and could easily hear a peculiar noise, and upon subsequent examination I was satisfied that she had ruptured her trachea by her injudicious straining. Pulse 110. Venesection to thirty ounces; two large basins of blood were lost before the breathing was relieved; pulse then 120 . She was ordered the following medicines :-

Sulphate of magnesia - . 4 drachms. Nitrate of potass . . . I drachm.

Potassio-tartrate of antimony . 2 grains. 